\title{
The evolution of the X-ray afterglow emission of GW 170817/ GRB 170817A in XMM-Newton observations
}

\author{
P. D’Avanzo ${ }^{1}$, S. Campana ${ }^{1}$, O. S. Salafia ${ }^{2,1,3,4}$, G. Ghirlanda ${ }^{1,2,3}$, G. Ghisellini ${ }^{1}$, A. Melandri ${ }^{1}$, \\ M. G. Bernardini ${ }^{5,1}$, M. Branchesi ${ }^{4,6}$, E. Chassande-Mottin ${ }^{7}$, S. Covino ${ }^{1}$, V. D’Elia ${ }^{8}$, L. Nava ${ }^{9,1}$, \\ R. Salvaterra ${ }^{10}$, G. Tagliaferri ${ }^{1}$, and S. D. Vergani ${ }^{11,1}$
}

\author{
${ }^{1}$ INAF - Osservatorio Astronomico di Brera, Via E. Bianchi 46, 23807 Merate (LC), Italy \\ e-mail: paolo.davanzo@brera.inaf.it \\ 2 Dipartimento di Fisica "G. Occhialini”, Università degli Studi di Milano-Bicocca, Piazza della Scienza 3, 20126 Milano, Italy \\ 3 INFN - Sezione di Milano-Bicocca, Piazza della Scienza 3, 20126 Milano, Italy \\ ${ }^{4}$ Gran Sasso Science Institute, Viale F. Crispi 7, L’Aquila, Italy \\ ${ }^{5}$ Laboratoire Univers et Particules de Montpellier, Université Montpellier, CNRS/IN2P3, Montpellier, France \\ ${ }^{6}$ INFN, Laboratori Nazionali del Gran Sasso, 67100 L'Aquila, Italy \\ ${ }^{7}$ APC, Université Paris Diderot, CNRS/IN2P3, CEA/Irfu, Obs de Paris, Sorbonne Paris Cité, Paris, France \\ ${ }^{8}$ Space Science Data Center, ASI, Via del Politecnico, s.n.c., 00133 Roma, Italy \\ 9 INAF, Osservatorio Astronomico di Trieste, Via G.B. Tiepolo 11, 34143 Trieste, Italy \\ ${ }^{10}$ INAF, Istituto di Astrofisica Spaziale e Fisica Cosmica di Milano, via E. Bassini 15, 20133 Milano, Italy \\ ${ }^{11}$ GEPI, Observatoire de Paris, PSL Research University, CNRS, Place Jules Janssen, 92190 Meudon, France
}

Received 18 January 2018 / Accepted 17 April 2018

\begin{abstract}
We report our observation of the short gamma-ray burst (GRB) GRB 170817A, associated to the binary neutron star merger gravitational wave (GW) event GW 170817, performed in the X-ray band with XMM-Newton $135 \mathrm{~d}$ after the event (on 29 December, 2017). We find evidence for a flattening of the X-ray light curve with respect to the previously observed brightening. This is also supported by a nearly simultaneous optical Hubble Space Telescope observation and successive X-ray Chandra and low-frequency radio observations recently reported in the literature. Since the optical-to-X-ray spectral slope did not change with respect to previous observations, we exclude that the change in the temporal evolution of the light curve is due to the passage of the cooling frequency: its origin must be geometric or dynamical. We interpret all the existing afterglow data with two models: i) a structured jet and ii) a jet-less isotropic fireball with some stratification in its radial velocity structure. Both models fit the data and predict that the radio flux must decrease simultaneously with the optical and X-ray emission, making it difficult to distinguish between them at the present stage. Polarimetric measurements and the rate of short GRB-GW associations in future LIGO/Virgo runs will be key to disentangle these two geometrically different scenarios.
\end{abstract}

Key words. gravitational waves - gamma rays: general

\section{Introduction}

A gravitational wave $(\mathrm{GW})$ event originated by the merger of a binary neutron star (BNS) system was detected for the first time by aLIGO/Virgo (GW 170817; Abbott et al. 2017a), and was found to be associated to the weak short gamma-ray burst (GRB) GRB 170817A detected by the Fermi and INTEGRAL satellites (Goldstein et al. 2017; Savchenko et al. 2017), marking the dawn of multi-messenger astronomy (Abbott et al. 2017b). The proximity of the event $41 \mathrm{Mpc}$; Hjorth et al. 2017, Cantiello et al. 2018) and the relative accuracy of the localisation $\left(\sim 30 \mathrm{deg}^{2}\right.$, thanks to the joint LIGO and Virgo operation) led to a rapid $(\Delta t<11 \mathrm{~h})$ identification of a relatively bright optical electromagnetic counterpart (EM), named AT2017gfo, in the galaxy NGC 4993 (Arcavi et al. 2017; Coulter et al. 2017; Lipunov et al. 2017; Melandri et al. 2017; Tanvir et al. 2017; Soares-Santos et al. 2017; Valenti et al. 2017). The analysis and modelling of the spectral characteristics of this source, together with their evolution with time, resulted in a good match with the expectations for a "kilonova" (i.e. the emission due to radioactive decay of heavy nuclei produced through rapid neutron capture; Li \& Paczyński 1998), providing the first compelling observational evidence for the existence of such elusive transient sources (Cowperthwaite et al. 2017; Drout et al. 2017; Evans et al. 2017; Kasliwal et al. 2017; Nicholl et al. 2017; Pian et al. 2017; Smartt et al. 2017; Villar et al. 2017). While the bright kilonova associated to GW 170817 has been widely studied and its main properties relatively well determined, the observations of the short GRB 170817A are more challenging for the current theoretical frameworks. Indeed, the properties of this short GRB appear puzzling in the context of observations collected over the past decades (Berger 2014; D’Avanzo 2015; Ghirlanda et al. 2015). The prompt $\gamma$-ray luminosity was significantly fainter (by a factor 2500 ) than typical short bursts (see, e.g. D'Avanzo et al. 2014). A faint afterglow was detected in the X-ray and radio bands only at relatively late times (starting from $\sim 9$ and $16 \mathrm{~d}$ after the GW/GRB trigger, respectively; Alexander et al. 2017; Haggard et al. 2017; Hallinan et al. 2017; Margutti et al. 2017; Troja et al. 2017a), while earlier 
observations provided only upper limits in these bands (Evans et al. 2017; Hallinan et al. 2017).

Similarly to long bursts, short GRBs are thought to be produced by a relativistic jet with a typical half-opening angle $\theta_{\text {jet }} \sim 5-15 \mathrm{deg}$ (Fong et al. 2016). However, whether or not BNS mergers can always efficiently produce a relativistic jet is still debated (Paschalidis et al. 2015; Ruiz et al. 2016; Kawamura et al. 2016). Given the small probability that our line of sight was within the jet half-opening angle, $1-\cos \left(\theta_{\text {jet }}\right)$, it is unlikely that the first short GRB associated to a GW event had a jet pointing towards the Earth. The extremely low $\gamma$-ray luminosity of GRB 170817A has been interpreted as being due to (i) the debeamed radiation of a jet observed off-beam (i.e. viewing angle $\theta_{\text {view }}>\theta_{\text {jet }}$ ), provided that the jet bulk Lorentz factor is significantly smaller than usually assumed (see, e.g. Pian et al. 2017). Alternatively, the jet could be (ii) structured, with a fast and energetic inner core surrounded by a slower, less energetic layer/sheath/cocoon (first proposed for long GRBs Lipunov et al. 2001; Rossi et al. 2002; Salafia et al. 2015 and only recently extended to short GRBs - Kathirgamaraju et al. 2018; Lazzati et al. 2017a; Gottlieb et al. 2017; Lazzati et al. 2017b; Lyman et al. 2018; Margutti et al. 2018; Troja et al. 2018a). In this scenario the faint, off-beam emission is due to the slower component, which originates from the interaction of the jet with the merger dynamical ejecta or the post-merger winds. Recently, Mooley et al. (2018) suggested the possibility that (iii) the jet was not successful in excavating its way through the ambient medium and that GRB 170817A was due to its vestige, a quasi-isotropic cocoon with a velocity profile. Last but not least, (iv) a jet-less interpretation of GRB 17017A could still be viable: an isotropic fireball, expanding ahead of the kilonova ejecta, which could account for both the low luminosity of the $\gamma$-ray event and the properties of the EM component in the radio and X-ray bands (Salafia et al. 2017). In this case, all BNS mergers should have this kind of faint, hard X-ray counterpart. All of the above scenarios have relatively clear predictions for the temporal and spectral evolution of the electromagnetic emission from X-rays to the radio band. A comprehensive discussion of the possible physical scenarios leading to the observed broad-band emission of GW 170817/GRB 170817A can be found in Nakar \& Piran (2018). Recent radio and X-ray observations (Mooley et al. 2018; Margutti et al. 2018; Ruan et al. 2018; Troja et al. 2018a), carried out until $\sim 110-115 \mathrm{~d}$ after the event, indicate that the source flux is steadily rising and that the spectral energy distribution (SED) over these bands is consistent with a single power-law component. These results disfavour interpretation (i) reported above (an off-beam homogeneous jet).

In this Letter we present deep, late-time X-ray observations of GW 170817/GRB 170817A carried out 135 days after the event with the XMM-Newton satellite, showing evidence for a a change in the temporal slope, indicating a flattening in the afterglow emission (Sect. 2). In Sect. 3 we interpret and discuss all the available afterglow data of GW 170817 / GRB 170817A under the structured jet and jet-less scenarios mentioned above and summarise our conclusions in Sect. 4.

\section{Observations and data analysis}

XMM-Newton started observing GW 170817 on 29 December 2017 at 19:00:11 UT, 134.5 d after the GW event. XMM-Newton observed for $41.3 \mathrm{ks}$ (42.8 ks) with the pn (MOS) detector, all equipped with the thin filter. Two large background flares occurred during the observation, reducing the usable time to

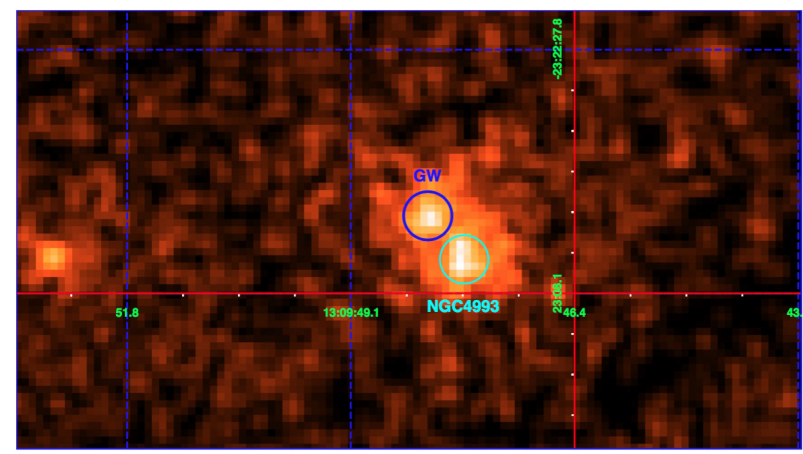

Fig. 1. X-ray image obtained by co-adding the XMM-Newton pn and MOS data presented in this paper. The X-ray emission of GW 170817/GRB 170817A (upper circle, 4" radius) is clearly visible close to the nucleus of its host galaxy NGC 4993 (lower circle).

26.0 and $29.6 \mathrm{ks}$ for the pn and MOS detectors, respectively. The centre of NGC 4993 lies at only 10" from GW 170817 (see Fig. 1) and particular care must be taken. We extracted products using a region of 4 " radius centred on the optical position of GW 170817/GRB 170817A (Coulter et al. 2017). The background has been extracted from two 4 " regions, at the same distance from the host galaxy centre, one opposite to GW 170817 and the other to the north-east (thus avoiding the source detected by Chandra, named "S2" in Margutti et al. (2018), in the southwest region). We gathered (source plus background) 60, 15 and 15 counts from the pn, MOS 1 and MOS 2 detectors, respectively, with the source making $70-80 \%$ of the total. Response matrices were generated with the package $S A S$ v16.1 using the latest calibration products.

Spectra were rebinned to five counts per spectral bin and C-statistics was adopted for the fits. We fit the three spectra with an absorbed power law model with the column density fixed to a Galactic value of $7.84 \times 10^{20} \mathrm{~cm}^{-2}$ (Kalberla et al. 2005). A 90\% confidence level (c.l.) upper limit on the intrinsic absorption is $<1.1 \times 10^{21} \mathrm{~cm}^{-2}$. The best fit provides a photon index $\Gamma=1.7_{-0.4}^{+0.5}(90 \%$ c.l.) and a $0.3-10 \mathrm{keV}$ unabsorbed flux $F_{X}=\left(2.1_{-0.8}^{+1.1}\right) \times 10^{-14} \mathrm{erg} \mathrm{cm}^{-2} \mathrm{~s}^{-1}$. This fit and its uncertainty region are shown in Fig. 2 by the dot-dashed line and the grey shaded region, respectively. The XMM-Newton de-absorbed data are also shown in Fig. 2 (blue points).

Motivated by the almost simultaneous XMM-Newton and HST observations ( $\sim 137 \mathrm{~d}$ after the event; Margutti et al. 2018), we dereddened ${ }^{1}$ the optical AB magnitude $\operatorname{mag}_{F 606 W}=26.90 \pm$ 0.25 reported by these authors and we fitted together optical and $\mathrm{X}$-ray data. Thanks to the large leverage in terms of energy range, we tightly constrain the overall power law photon index to $\Gamma=$ $1.60 \pm 0.05$. This fit is shown by the dashed red line and its uncertainty by the yellow shaded region in Fig. 2. Adopting this index in the range $0.3-10 \mathrm{keV}$, the unabsorbed flux is $F_{X}=\left(2.1_{-0.5}^{+0.7}\right) \times$ $10^{-14} \mathrm{erg} \mathrm{cm}^{-2} \mathrm{~s}^{-1}$, which translates to a luminosity $L_{X}=4 \times$ $10^{39} \mathrm{erg} \mathrm{s}^{-1}$ (at $41 \mathrm{Mpc}$ ). The XMM-Newton flux and photon index are fully consistent with the values found about one month before and after our observation by Chandra (namely, a $0.3-$ $10 \mathrm{keV}$ unabsorbed flux $F_{X}=(2.5 \pm 0.3) \times 10^{-14} \mathrm{erg} \mathrm{cm}^{-2} \mathrm{~s}^{-1}$ and $F_{X}=(2.6 \pm 0.3) \times 10^{-14} \mathrm{erg} \mathrm{cm}^{-2} \mathrm{~s}^{-1}$ measured at $\sim 109$ and 159 days after the GW trigger, respectively; Margutti et al. 2018; Troja et al. 2018a,b) and indicate that the GW 170817 flux stopped increasing.

1 We corrected for Galactic extinction assuming $E(B-V)=0.105$ mag (Schlafly \& Finkbeiner 2011). 


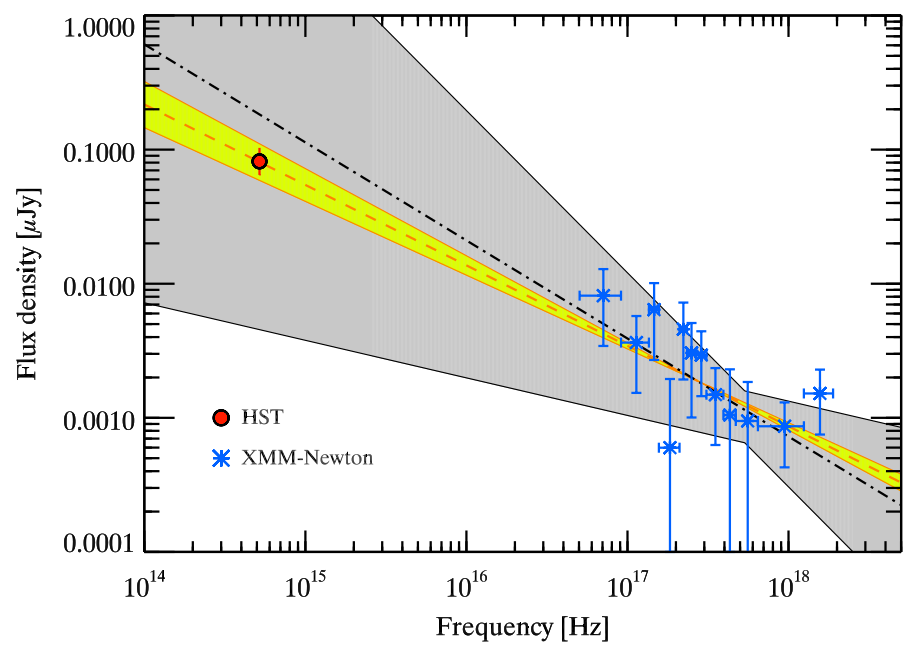

Fig. 2. Optical to X-ray spectrum of GRB 170817A. XMM-Newton data points (blue, this work) and $H S T$ contemporaneous detection (red circle) are shown. The grey shaded region shows the $90 \%$ uncertainty on the fit of the XMM-Newton data alone (dot-dashed line). The fit obtained combining the de-reddened HST flux (from Margutti et al. 2018) and our XMM-Newton data results in a photon index of 1.6 (dotted red line) with an error of \pm 0.05 (90\% c.l. - yellow shaded region).

\section{Interpretation and discussion}

Figure 3 shows the afterglow data published in the literature to date, together with our XMM-Newton point obtained at $\sim 135 \mathrm{~d}$ (light blue circle). All radio and X-ray detections of GRB 170817A reported so far covering the time range between $\sim 9$ and $\sim 115 \mathrm{~d}$ after the event indicated a steady increase of the source flux $\left(F(t) \propto t^{0.7-0.8}\right)$, with negligible spectral evolution over a broadband spectrum $\left(F_{v} \propto v^{-0.6}\right.$; Mooley et al. 2018; Margutti et al. 2018; Ruan et al. 2018; Troja et al. 2018a). By comparing the nearly simultaneous XMM-Newton and HST observations (see Sect. 2) we find that the SED at the epoch of our XMMNewton observation is still consistent with a single power-law component between the X-ray and the optical, with $F_{v} \propto v^{-0.6}$ (Fig. 2). Given the lack of spectral evolution, we found it reasonable to assume that the light curve is evolving in the same way at all wavelengths and carried out a combined fit of the available radio $(3 \mathrm{GHz})$, optical ${ }^{2}$ and X-ray $(0.3-10 \mathrm{keV})$ data obtained between $\sim 9$ and $\sim 159 \mathrm{~d}$ after the event (Hallinan et al. 2017; Mooley et al. 2018; Lyman et al. 2018; Margutti et al. 2018; Troja et al. 2018a,b). Using an F-test we compared the joined fit obtained with a single and a broken power-law model. We find that a temporal break is required at $1.94 \sigma(95 \%$ c.l. $)$. While with a single power-law $\left(F(t) \propto t^{\alpha}\right)$ model we obtain an index $\alpha \sim 0.8$, in agreement with other findings (Mooley et al. 2018; Margutti et al. 2018; Troja et al. 2018a), at the same time we note that both the XMM-Newton (this work) and Chandra ${ }^{3}$ (Troja et al. 2018b) data points obtained at successive epochs over two months fall below the extrapolation of the best fit (by 1.5 and 2.7 sigma, respectively). Such an increasing divergence, together with the

2 Concerning the optical band, we included in our fit only the HST data ( $F 606 W$ filter) obtained at $\sim 110$ and $\sim 137 \mathrm{~d}$ after the $\mathrm{GW}$ trigger, that is, those obtained when the thermal component due to the kilonova emission is no longer contributing (Lyman et al. 2018; Margutti et al. 2018).

3 A different result, with a X-ray light curve consistent with a $t^{0.7}$ rise, is found by Haggard et al. (2018), based on the same Chandra observations used by Troja et al. (2018b), although these authors reported their results in a slightly different energy band. mild indication of a temporal break provided by the F-test, is indicative of a change in the slope, with a flattening, of the X-ray light curve with respect to the brightening trend observed in the $\mathrm{X}$-ray and radio bands at earlier epochs. This change in the light curve temporal evolution is observed also in the optical band by the HST observations carried out at $\sim 110$ and $\sim 137 \mathrm{~d}$ with $H S T$ (Lyman et al. 2018; Margutti et al. 2018; see also Fig. 3). Furthermore, the evidence for a possible plateau in the light curve has recently been found in low-frequency radio data obtained between 66 and 152 days after the event (Resmi et al. 2018), although the relatively poor temporal sampling prevents us from firmly excluding a rising trend. Overall, the multi-wavelength behaviour of the afterglow provides an indication that the light curve is changing slope (see also Dobie et al. 2018), even if it is too early to constrain the temporal evolution after the break, since at this epoch we are still sampling its turning point.

The X-ray spectrum expected if the cooling frequency has transitioned below the X-ray band (between the earlier Chandra observation and our XMM-Newton epoch) has a photon index of 2.1 and predicts an optical flux that is inconsistent with that observed by HST at almost the same epoch. We can therefore exclude this explanation of the observed optical and X-ray light curve peak. We rather interpret it as being due to a dynamical or geometric effect. We interpret the optical and X-ray light curve within two possible scenarios: (i) a structured jet, in which case the decline of the optical and X-ray fluxes indicates that the emission from the jet core has entered our line of sight (i.e. the core has decelerated down to a Lorentz factor $\Gamma \sim \theta_{\text {view }}^{-1}$ ); or (ii) an isotropic (Salafia et al. 2017) and stratified fireball with a velocity profile, like that described in Mooley et al. (2018), in which case the light curve peak indicates that the velocity profile has a rather sharp cut-off at $\beta_{\min }=v_{\min } / c \sim 0.88$.

\subsection{The structured jet model}

If a jet is launched after the merger, it must excavate its way out of the inner region, which can be baryon-polluted due to the postmerger winds and the dynamical ejecta. The propagation through such ambient material is likely to play a major role in shaping the jet angular distribution of energy and terminal Lorentz factor at breakout (see e.g. the simulations by Lazzati et al. 2017b). The resulting jet structure features an inner, narrow, faster core with a relatively uniform distribution of kinetic energy per unit solid angle, surrounded by a slower, extended structure whose kinetic energy per unit solid angle decreases relatively quickly with the distance from the jet axis. This latter structure can be identified as the vestige of the jet cocoon (constituted by the jet and ambient material that has been shocked during the excavation). Guided by this picture, we employ a simple structured jet model, in which both the isotropic equivalent kinetic energy $E_{\mathrm{K} \text {,iso }}$ and the bulk Lorentz factor $\Gamma$ are approximately constant within a narrow core of half-opening angle $\theta_{\text {core }}$ and decrease as power-laws outside of it:

$E_{\mathrm{K}, \text { iso }}(\theta)=\frac{E_{\mathrm{K}, \text { iso,core }}}{1+\left(\theta / \theta_{\text {core }}\right)^{s_{1}}}$,

and

$\Gamma_{0}(\theta)=1+\frac{\Gamma_{\text {core }}-1}{1+\left(\theta / \theta_{\text {core }}\right)^{s_{2}}}$.

We model the dynamics of the jet with the simplifying assumption that each solid angle element evolves independently (i.e. we neglect side expansion, which should have a limited effect 
Table 1. Parameters of the structured jet and isotropic outflow models shown in Fig. 3. Units in square parentheses.

\begin{tabular}{lr|lr}
\hline \hline \multicolumn{2}{c|}{ Structured jet } & \multicolumn{2}{c}{ Isotropic outflow } \\
\hline$\theta_{\text {core }}[\mathrm{deg}]$ & 2 & & \\
$E_{\mathrm{K}, \text { iso,core }}[\mathrm{erg}]$ & $1 \times 10^{52}$ & $E_{0}[\mathrm{erg}]$ & $3 \times 10^{51}$ \\
$s_{1}$ & 3.5 & $\alpha$ & 5 \\
$\Gamma_{\text {core }}$ & 110 & $\Gamma_{\max }$ & 3.8 \\
$s_{2}$ & 2 & $\beta_{\text {min }}$ & 0.875 \\
$\theta_{\text {view }}$ & 22.5 & & \\
$n\left[\mathrm{~cm}^{-3}\right]$ & $10^{-3}$ & $n\left[\mathrm{~cm}^{-3}\right]$ & $2 \times 10^{-4}$ \\
$\epsilon_{\mathrm{e}}$ & 0.06 & $\epsilon_{\mathrm{e}}$ & 0.1 \\
$\epsilon_{\mathrm{B}}$ & 0.01 & $\epsilon_{\mathrm{B}}$ & 0.01 \\
$p$ & 2.13 & $p$ & 2.14 \\
\hline
\end{tabular}

on the light curve, see e.g. Granot \& Kumar 2003; Lazzati et al. 2017b; Lamb \& Kobayashi 2017). For each solid angle element, we model the emission following Sari et al. (1998), with the proper transformations to the off-axis observer frame. The ambient medium is assumed to have a constant number density $n$. The parameters of the structured jet model shown in Fig. 3 (dashed lines) are reported in Table 1 (where $\epsilon_{e}$ and $\epsilon_{B}$ are the shock energy carried by the electrons and by the magnetic field, respectively, and $p$ is the electron energy distribution index). With the given parameters, the total kinetic energy in the jet is $E_{\text {jet }} \approx 1.1 \times 10^{49} \mathrm{erg}$ (for one jet), which is just what is expected for a standard short GRB jet (Hotokezaka et al. 2016). Different structured jet scenarios have been proposed to model the afterglow light curves of GRB 170817A by Lazzati et al. (2017b), Lyman et al. (2018), Margutti et al. (2018) and Troja et al. (2018a). As in our case described above, the models presented in Lyman et al. (2018), Margutti et al. (2018) and Troja et al. (2018a) can account for the change in the slope observed in the X-ray and optical light curve at $t \sim 110-130$ $\mathrm{d}$, predicting a relatively long plateau at these epochs. We note, however, that all the proposed models are very similar and that the diversity in the predictions can be ascribed to a combination of differences in the jet structure (including the opening angle of the relativistic core) and the density of the environment, and to the different choice of the microphysical parameters, that can be better constrained with future multi-band observations.

\subsection{The isotropic outflow model}

Salafia et al. (2018) proposed a scenario where a reconnectionpowered isotropic fireball is launched at the beginning of the neutron-star merger phase. The simple model sketched there assumed a uniform energy profile in the fireball; however, the described process may also produce a fireball with an energy profile like that described in Mooley et al. (2018). In what follows, we adopt a similar model to that in Mooley et al. (2018) to describe the light curve in our jet-less scenario, with the difference that we take into account the proper equal-arrival-time surfaces in the computation of the observed flux. In this scenario, an isotropic (or quasi-isotropic) outflow is launched, with a distribution of energy in momentum space given by:

$E(>\Gamma \beta)=E_{0}(\Gamma \beta)^{-\alpha}$,

between the minimum and maximum Lorentz factors $\Gamma_{\min }, \Gamma_{\max }$ or equivalently the minimum and maximum velocities $\beta_{\min }, \beta_{\max }$. The interaction with the ISM results in a shock whose dynamics
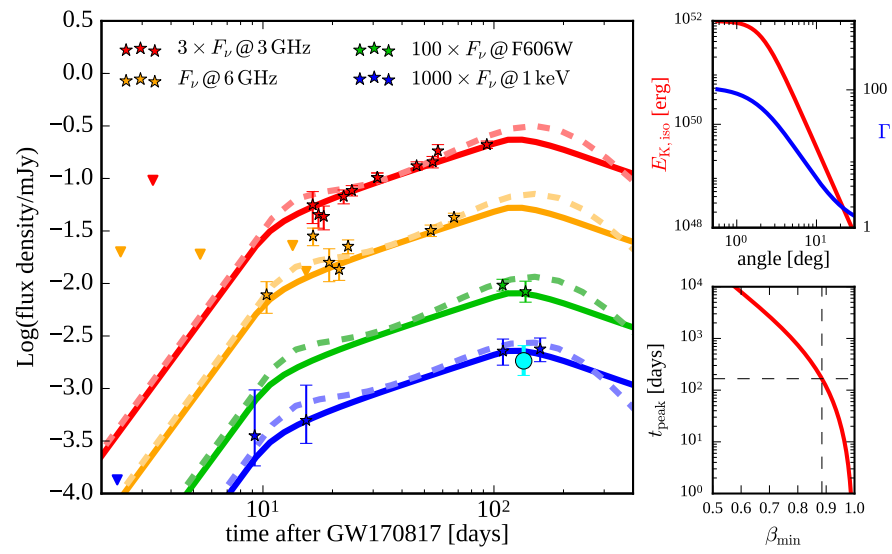

Fig. 3. Left-hand panel: GRB 170817A afterglow light curves in radio at $3 \mathrm{GHz}$ and $6 \mathrm{GHz}$ (red and orange stars, respectively, VLA observations - data from Hallinan et al. 2017; Mooley et al. 2018), in the optical (green stars, HST/ACS observations in the F606W filter - data from Lyman et al. 2018 and Margutti et al. 2018) and in the X-rays (blue stars: Chandra observations, data from Margutti et al. 2018; Troja et al. 2018b; light blue circle: our XMM-Newton observation). Thick coloured solid lines represent our isotropic fireball model (corresponding to either the jet-less scenario outlined in Salafia et al. 2017, or the choked jet scenario proposed by Mooley et al. 2018). The brown dashed lines represent our structured jet model. The parameters of both models are reported in Table 1. Upper right-hand panel: the jet structure assumed in our model. The red line represents the isotropic equivalent kinetic energy, while the blue line shows the Lorentz factor. Lower right-hand panel: the red line shows the peak time of the isotropic outflow light curve as a function of the minimum velocity $\beta_{\min }$ in the velocity profile. The dashed lines mark the value we employed in the modelling.

reflect the fact that slower (but more energetic) ejecta progressively cross the reverse shock, reducing the deceleration. The evolution of the forward shock radius (Hotokezaka et al. 2016) is given by

$$
\frac{4}{3} \pi R^{3} m_{p} n(c \beta \Gamma)^{2}=E(>\Gamma \beta)
$$

where $m_{p}$ is the proton mass. As soon as all the outflow material has gone across the reverse shock, that is, after the minimum ejecta velocity $\beta_{\min }$ has been reached, the dynamics turn into simple adiabatic expansion, with $\Gamma \beta \propto R^{-3 / 2}$ (Nava et al. 2013). We model the synchrotron emission from the shock-heated electrons following Sari et al. (1998), just as in the structured jet model. The parameters are essentially the same as in Mooley et al. (2018), except for the slightly lower value of the electron energy power law slope $p=2.14$, which provides a better agreement to the broadband spectrum (see e.g. Margutti et al. 2018 therein Fig. 6), and for the introduction of the minimum ejecta velocity $\beta_{\min }$ in order to account for the peak in the light curve. The parameters of the isotropic outflow model shown in Fig. 3 (solid lines) are reported in Table 1 . The value $\beta_{\min }=0.875$ we employed in the modelling implies a total kinetic energy $E_{\text {tot }} \approx 1.6 \times 10^{50} \mathrm{erg}$ (assuming spherical geometry).

\section{Conclusions}

The XMM-Newton late-time observations of the afterglow of GRB 170817A associated to the BNS merger event GW 170817 presented in this work show evidence that the X-ray flux had flattened during the last two months of data collection (Dec 2017-Jan 2018). This is supported by the latest HST observations 
at the same epoch (Margutti et al. 2018), by later Chandra X-ray observations (Troja et al. 2018b) and by late-time GMRT lowfrequency radio observations (Resmi et al. 2018). The combined spectrum obtained with nearly simultaneous XMM-Newton and $H S T$ data show no spectral evolution with respect to previous observations, suggesting a geometric or dynamical origin for the decrease in flux observed in the afterglow light curve. We modelled the observed X-ray, optical, and radio afterglow emission as (i) the deceleration peak of the core of a structured jet (as described in Sect 3.1) pointing away from our line of sight or (ii) the deceleration of an isotropic fireball with a radial velocity structure. We found that both models successfully reproduce the available data, which is not surprising since in both cases we are still observing the emission from the slower ejecta. The similarity of the light curves of the two models as shown in Fig. 3 may require some independent measurements to disentangle between these two possible scenarios. A possible diagnostic test able to discriminate between isotropic and jetted geometries is based on linear polarisation measurements, given that, to observe polarisation, some degree of asymmetry is needed (Rossi et al. 2004; Covino \& Gotz et al. 2016 and references therein). A general prediction for late-time afterglows is that they can show linear polarisation even up to a fairly high level $(\sim 10 \%)$ depending on the assumed geometry (see Rossi et al. 2004 for more details). With the presently available technologies, for the AT2017gfo at late times, such a measurement is very demanding and could only be feasible (in the most optimistic case, i.e. for polarisation levels of $5-10 \%$ ) at radio wavelengths ${ }^{4}$. On the contrary, no polarisation is essentially expected for an isotropic emission. However, while detection of linear polarisation would be a clear indication of a jetted geometry, a null result may not be conclusive. At radio frequencies, linear polarisation can be detected at frequencies higher than the self-absorption frequency but it can also be suppressed by Faraday rotation depending on the specific micro-physical parameters (Toma et al. 2008), making the interpretation of null polarisation difficult and possibly inconclusive $^{5}$ without meaningful observations at higher frequencies. Besides this, such a different geometry is expected to significantly affect the rate of burst, similar to GRB 170817A observed in association with GW events detected during the forthcoming LIGO/Virgo observing runs (Ghirlanda et al., in prep.).

Acknowledgements. We thank Norbert Schartel and the XMM-Newton staff for approving, scheduling and carrying out these observations. We thank A. Possent for useful discussion. We acknowledge support from ASI grant I/004/11/3. MGB acknowledges support of the OCEVU Labex (ANR-11-LABX-0060) and the A*MIDEX project (ANR-11-IDEX-0001-02) funded by the "Investissements d'Avenir" French government program managed by the ANR

\section{References}

Abbott, B. P., Abbott R., Abbott T. D., et al. 2017a, Phys. Rev. Lett., 119, 161101 Abbott, B. P., Abbott R., Abbott T. D., et al. 2017b, ApJ, 848, L12 Alexander, K. D., Berger, E., Fong, W., et al. 2017, ApJ, 848, L21 Arcavi, I., Hosseinzadeh, G., Howell, D. A., et al. 2017, Nature, 551, 64 Berger, E. 2014, ARA\&A, 52, 43

Cantiello, M., Jensen, J. B., Blakeslee, J. P., et al. 2018, ApJ, 854, L31 Coulter, D. A., Foley, R. J., Kilpatrick, C. D., et al. 2017, Science, 358, 1556 Covino, S., \& Gotz, D. 2016, Astron. Astrophys. Trans., 29, 205

Cowperthwaite, P. S., Berger E., Villar V. A., et al. 2017, ApJ, 848, L17

D'Avanzo, P., 2015, JHEAp, 7, 73

\footnotetext{
4 Optical polarisation studies should in principle be discriminant as well, but the source is too faint in this band.

5 Further discussion and predictions about radio polarimetry of GW 170817 have recently been reported by Geng et al. (2018) and Gill et al. (2018).
}

D’Avanzo, P., Salvaterra, R., Bernardini, M. G., et al. 2014, MNRAS, 442, 2342 Dobie, D., Salvaterra, R., Bernardini, M. G., et al. 2018, ArXiv e-prints [arXiv: 1803.06853]

Drout, M. R., Piro, A. L., Shappee, B. J., et al. 2017, Science, 358, 1570 Evans, P. A., Cenko, S. B., Kennea, J. A., et al. 2017, Science, 358, 1565 Fong, W., Margutti, R., Chornock, R., et al. 2016, ApJ, 833, 151 Geng, J., Dai, Z.-G., Huang, Y.-F., et al. 2018, ApJ, 856, L33

Ghirlanda, G., Bernardini, M. G., Calderone, G., \& D’Avanzo, P. 2015, JHEAp, 7,81

Gill, R., \& Granot, J. 2018, MNRAS, submitted [arXiv:1803.05892]

Granot, J., \& Kumar, P. 2003, ApJ, 591, 1086

Granot, J., Panaitescu, A., Kumar, P., \& Woosley, S. E. 2001, ApJ, 570, L61

Goldstein, A., Veres P., von Kienlin A., et al. 2017, ApJ, 848, L14

Gottlieb, O., Nakar, E., Piran, T., \& Hotokezaka, K. 2017, ArXiv e-prints [arXiv: 1710.05896]

Haggard, D., Nynka, M., Ruan, J. J., et al. 2017, ApJ, 848, L25

Haggard, D., Nynka, M., Ruan, J. J., et al. 2018, GRB Coordinates Network, 22371

Hallinan, G., Corsi, A., Mooley, K. P., et al. 2017, Science, 358, 1579

Hjorth, J., Levan, A. J., Tanvir, N. R., et al., 2017, ApJ, 848, L31

Hotokezaka, K., Nissanke, S., Hallinan, G., et al. 2016, ApJ, 831, 190

Kalberla, P. M., Burton, P. M. W., Hartmannet W. B., et al. 2005, A\&A, 440, 775

Kasliwal, M. M., Nakar, E., Singer, L. P., et al. 2017, Science, 358, 1559

Kathirgamaraju, A., Barniol Duran, R., \& Giannios, D. 2018, MNRAS, 473, L121

Kawamura, T., Giacomazzo, B., Kastaun, W., et al. 2016, Phys. Rev. D, 94, 4012

Lamb, G. P., \& Kobayashi, S., 2017, MNRAS, 472, 4953

Lazzati, D., Deich, A., Morsony, B. J., \& Workman, J. C. 2017a, MNRAS, 471, 1652

Lazzati, D., Perna, R., Morsony, B. J., et al. 2017b, ArXiv e-prints [arXiv: 1712.03237]

Li, L.-X., \& Paczyński, B. 1998, ApJ 507, 59

Lipunov, V. M., Postnov, K. A., \& Prokhorov, M. E. 2001, Astron. Rep., 45, 236

Lipunov, V. M., Gorbovskoy, E., Kornilov, V. G., et al. 2017, ApJ, 850, 1

Lyman, J. D., Lamb, G. P., Levan, A. J., et al. 2017, ArXiv e-prints [arXiv: 1801.02669]

Margutti, R., Berger, E., Fong, W., et al. 2017, ApJ, 848, L20

Margutti, R., Alexander, K. D., Xie, X., et al. 2018, ApJ, 856, L18

Melandri, A., Campana, S., Covino, S., et al. 2017, GRB Coordinates Network, 21532

Mooley, K., Nakar, E., Hotokezaka, K., et al. 2018, Nature, 554, 207

Nakar, E., \& Piran, T. 2018, ArXiv e-prints [arXiv:1801. 09712]

Nava, L., Sironi, L., Ghisellini, G., et al. 2013, MNRAS, 433, 2107

Nicholl, M., Berger, E., Kasen, D., et al. 2017, ApJ, 848, L18

Paschalidis, V., Ruiz, M., \& Shapiro, S. L. 2015, ApJ, 806, 14

Pian, E., D'Avanzo, P., Benetti, S., et al. 2017, Nature, 551, 67

Resmi, L., Schulze, S., Ishwara Chandra, C. H., et al., 2018, ApJ, submitted [arXiv: 1803.02768]

Rossi, E., Lazzati, D., \& Rees, M. J. 2002, MNRAS, 332, 945

Rossi, E., Lazzati, D., Salmonson, J. D., \& Ghisellini, G., 2004, MNRAS, 354, 86

Ruan, J. J., Nynka, M., Haggard, D., et al., 2018, ApJ, 853, L4

Ruiz, M., Lang, R. N., Paschalidis, V., \& Shapiro, S. L. 2016, ApJ, 824, 6

Salafia, O. S., Ghisellini, G., Pescalli, A., Ghirlanda, G., \& Nappo, F. 2015, MNRAS, 450, 3549

Salafia, O. S., Ghisellini, G., Ghirlanda, G., \& Colpi, M. 2017, A\&A, submitted [arXiv:1711.03112]

Salafia, O. S., Ghisellini, G., \& Ghirlanda, G., 2018, MNRAS, 474, L7

Sari, R., Piran, T., and Narayan, R., 1998, ApJ, 497, L17

Savchenko, V., Bazzano A., Bozzo E., et al. 2017, ApJ, 848, L15

Schlafly, E. F., \& Finkbeiner, D. P., 2011, ApJ, 737, 103

Smartt, S. J., Chen, T.-W., Jerkstrand, A., et al. 2017, Nature, 551, 75

Soares-Santos, M., Holz, D. E., Annis, J., et al. 2017, ApJ, 848, L16

Tanvir, N. R., Levan, A. J., González-Fernández, C., et al. 2017, ApJ, 848, 27

Toma, K., Ioka, K., Nakamura, T., Ioka, K., \& Nakamura, T. 2008, ApJ, 673, L123

Troja, E., Piro, L., van Eerten, H., et al. 2017a, Nature, 551, 71

Troja, E., Piro L., Ryan, G., et al. 2017b, GRB Coordinates Network, 22201

Troja, E., Piro, L., Ryan, G., et al. 2018a, MNRAS, in press

Troja, E., Piro, L., van Eerten, H., et al. 2018b, GRB Coordinates Network, 22374

Valenti, S., David, J. S., Yang, S., et al. 2017, ApJ, 848, 24

Villar, V. A., Guillochon, J., Berger, E., et al. 2017, ApJ, 851, 21 